\title{
As Long as You Name My Name Right: Social Circles and Social Sentiment in the Hollywood Hearings
}

\author{
Oren Tsur ${ }^{\dagger \S}$ \\ Dan Calacci ${ }^{\dagger}$ \\ orentsurdseas.harvard.edu dcalacci@ccs.neu.edu \\ David Lazer ${ }^{\dagger \star}$ \\ d.lazer@neu.edu \\ ${ }^{\dagger}$ Lazer Laboratory, Northeastern University \\ ${ }^{\S}$ School of Engeneering and Applied Sciences, Harvard University \\ ${ }^{\star}$ Harvard Kennedy School, Harvard University
}

\begin{abstract}
The Hollywood Blacklist was based on a series of interviews conducted by the House Committee on Un-American Activities (HUAC), trying to identify members of the communist party. We use various NLP algorithms in order to automatically analyze a large corpus of interview transcripts and construct a network of the industry members and their "naming" relations. We further use algorithms for Sentiment Analysis in order to add a psychological dimension to the edges in the network. In particular, we test how different types of connections are manifested by different sentiment types and attitude of the interviewees. Analysis of the language used in the hearings can shed new light on the motivation and role of network members.
\end{abstract}

\section{Introduction}

A growing body of computational research is focused on how language is used and how it shapes/is shaped by a community of speakers. Computational works in the nexus of language and the social arena deal with various topics such as language accommodation (Danescu-NiculescuMizil and Lee, 2011; Danescu-Niculescu-Mizil et al., 2011), demographic language variation (Eisenstein et al., 2010; O'Connor et al., 2010), the factors that facilitate the spread of information in Q\&A forums and social networks (Adamic et al., 2008; Bian et al., 2009; Romero et al., 2011) or the correlation between words and social actions (Adali et al., 2012).

All of these works analyze the language and the social dynamics in online communities, mainly due to the increasing popularity of online social networks and greater availability of such data.

However, large scale socio-linguistic analysis should not be restricted to online communities and can be applied in many social and political settings beyond the online world. Two examples are the study of power structures in arguments before the U.S. Supreme Court (Danescu-Niculescu-Mizil et al., 2012) and the evolution of specific words and phrases over time as reflected in Google Books (Goldberg and Orwant, 2013).

In this paper we propose using network science and linguistic analysis in order to understand the social dynamics in the entertainment industry during one of its most controversial periods - the 'red scare' and the witch hunt for Communists in Hollywood during 1950's.

Historical background The Hollywood hearings (often confused with Senator McCarthy's hearings and allegations) were a series of interviews conducted by the House Committee on UnAmerican Activities (HUAC) in the years 19471956. The purpose of the committee was to conduct "hearings regarding the communist infiltration of the motion picture industry" (from the HUAC Annual Report). The committee subpoenaed witnesses such as Ayn Rand (writer), Arthur Miller (writer), Walt Disney (producer), future U.S. president Ronald Reagan (Screen Actors Guild), Elia Kazan (writer, actor, director) and Albert Maltz (Screen Writers Guild). Some of the witnesses were 'friendly' while some others were uncooperative ${ }^{1}$, refusing to "name names" or self incriminate ${ }^{2}$. Those who were named and/or were uncooperative were often jailed or effectively lost their job.

Arguably, many friendly witnesses felt they were complying with their patriotic duty. Many

\footnotetext{
${ }^{1}$ A note about terminology: by using the terms friendly and uncooperative there is no implied moral judgment - these are the terms used in the literature.

${ }^{2}$ It should be noted that being a member of the Communist party was not illegal, however, some individuals avoided self "incrimination" either in an effort to protect their job or as an ideological declaration in favor of privacy protection as a civil right protected by the constitution.
} 
others were threatened or simply manipulated to name names, and some later admitted to cooperating for other reasons such as protecting their work or out of personal vendettas and professional jealousies. It is also suspected that some naming occurred due to increasing professional tension between some producers and the Screen Writers Guild or (Navasky, 2003).

Motivation In this work we analyze a collection of HUAC hearings. We wish to answer the following questions:

1. Do sentiment and other linguistic categories correlate with naming relations?

2. Can we gain any insight on the social dynamics between the people in the network?

3. Does linguistic and network analysis support any of the social theories about dynamics at Hollywood during that time?

In order to answer the questions above we build a social graph of members of the entertainment industry based on the hearings and add sentiment labels on the graph edges. Layering linguistic features on a the social graph may provide us with new insights related to the questions at hand. In this short paper we describe the research framework, the various challenges posed by the data and present some initial promising results.

\section{Data}

In this work we used two types of datasets: Hearing Transcripts and Annual Reports. Snippets from hearings can be found in Figures 1(a) and 1(b), Figure 1(c) shows a snippet from an annual report. The transcripts data is based on 47 interviews conducted by the HUAC in the years 19512. Each interview is either a long statement (1(a)) or a sequence of questions by the committee members and answers by a witness (1(b)). In total, our hearings corpus consists of 2831 dialogue acts and half a million words.

\section{Named Entity Recognition and Anaphora Resolution}

The snippets in Figure 1 illustrates some of the challenges in processing HUAC data. The first challenge is introduced by the low quality of the available documents. Due to the low quality of
For the approximately 19 months of my membership, I was assigned to a "unit" Fed of those party memlers who were, like misself, members of the Group Theatre acting company. These were--

J Eimaril Bromberg, co-leader of the unit, deceaset.

Phebe Prand (Iater MIrs Morris Carnorsks) I was instrumental in bringing her into the party.

Aorris Carnorsky wh wellman (see below), lie racruted me into he narty

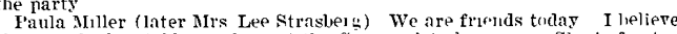
that. as she has told me, she quit the Communists long ago She is far to a man, to have remanted ausing them
Clifford Odets' He has assured me that he got out ahout the same time I did. Art Smith.

(a) A snippet from the testimony of Elia Kazan, (actor, writer and director, 3 times Academy Awards winner), 4.10.1952. Mr. TaVENNer. When you first became a member of the Communist
Party, did you meet an individual bv the name of Stanley Lawrence? Mr. Astr. I met Mr. Lawrence later. Mr. Lawrence came along in about 1935. He was misrepresented to the Communist Party as being an expert in underground work and that he was a liaison man in I an , $\mathrm{T}$ belise . Com Angeles taxicab driver.

Mr. TaneNNen. That was a device used by the leadership of the

to use him to educate the party leadership in Los Angeles County on underground work and things of that

(b) A snippet from the testimony of Harold Ashe's (journalist) testimony 9.17 19.1951

ANNUAL REPORT, COMMITTEE ON UN-AMERICAN ACTIVITIES 29

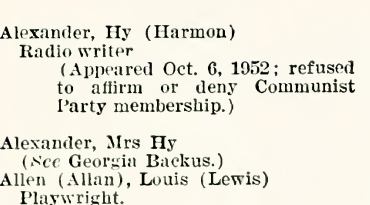

(c) A snippet from 1951 annual report.

Figure 1: Snippets from HUAC hearings and an annual report.

the documents the OCR output is noisy, containing misidentified characters, wrong alignment of sentences and missing words. These problems introduce complications in tasks like named entity recognition and properly parsing sentences.

Beyond the low graphic quality of the documents, the hearings present the researcher with the typical array of NLP challenges. For example, the hearing excerpt in 1(b) contains four dialogue acts that need to be separated and processed. The committee member (Mr. Tavenner) mentions the name Stanley Lawrence, later referred to by the witness (Mr. Ashe) as Mr. Lawrence and he thus coreference resolution is required before the graph construction and the sentiment analysis phases.

As a preprocessing stage we performed named entity recognition (NER), disambiguation and unification. For the NER task we used the Stanford NER (Finkel et al., 2005) and for disambiguation and unification we used a number of heuristics based on edit distance and name distribution. 
We used the Stanford Deterministic Coreference Resolution System (Lee et al., 2011) to resolve anaphoric references.

\section{Naming Graph vs. Mentions Graph}

In building the network graph of the members of the entertainment industry we distinguish between mentioning and naming in our data. While many names may be mentioned in a testimony (either by a committee member or by the witness, see example in Figures 1(a) and 1(b)), not all names are practically 'named' (=identified) as Communists. We thus use the hearings dataset in order to build a social graph of mentions (MG) and the annual reports are used to build a naming graph (NG). The NG is used as a "gold standard" in the analysis of the sentiment labels in the MG. Graph statistics are presented in Table 1.

While the hearings are commonly perceived as an "orgy of informing" (Navasky, 2003), the difference in network structure of the graphs portrays a more complex picture. The striking difference in the average out degree suggests that while many names were mentioned in the testimonies (either in a direct question or in an answer) - majority of the witnesses avoided mass-explicit naming ${ }^{3}$. The variance in outdegree suggests that most witnesses did not cooperate at all or gave only a name or two, while only a small number of witnesses gave a long list of names. These results are visually captured in the intersection graph (Figure 2) and were also manually verified.

The difference between the MG and the NG graph in the number of nodes with out-going edges (214 vs. 66) suggests that the HUAC used other informers that were not subpoenaed to testify in a hearing ${ }^{4}$.

In the remainder of this paper we analyze the the distribution of the usage of various psychological categories based on the role the witnesses play.

\section{Sentiment Analysis and Psychological Categories}

\subsection{Sentiment Analysis}

We performed the sentiment analysis in two different settings: lexical and statistical. In the lexi-

\footnotetext{
${ }^{3}$ Ayn Rand and Ronald Reagan, two of the most 'friendly' witnesses (appeared in front of the HUAC in 1947), did not name anyone.

${ }^{4}$ There might be some hearings and testimonies that are classified or still not publicly accessible.
}

\begin{tabular}{|c|c|c|c|}
\hline & MG & NG & Intersection \\
\hline Num of nodes & 1353 & 631 & 122 \\
\hline Num of edges & 2434 & 842 & 113 \\
\hline Nodes / Edges & 0.55 & 0.467 & 1 \\
\hline Avg. out degree & 36.87 & 3.93 & 8.7 \\
\hline Avg. in degree & 1.82 & 1.83 & 1.04 \\
\hline Var(outdegree) & 3902.62 & 120.75 & 415.59 \\
\hline Var(indegree) & 4.0 & 2.51 & 1.04 \\
\hline Nodes with out going edges & 66 & 214 & 13 \\
\hline Nodes with incoming edges & 1341 & 459 & 109 \\
\hline Reciprocity & 0.016 & 0.012 & 0 \\
\hline
\end{tabular}

Table 1: Network features of the Mentions graph, the Naming graph and the intersection of the graphs.

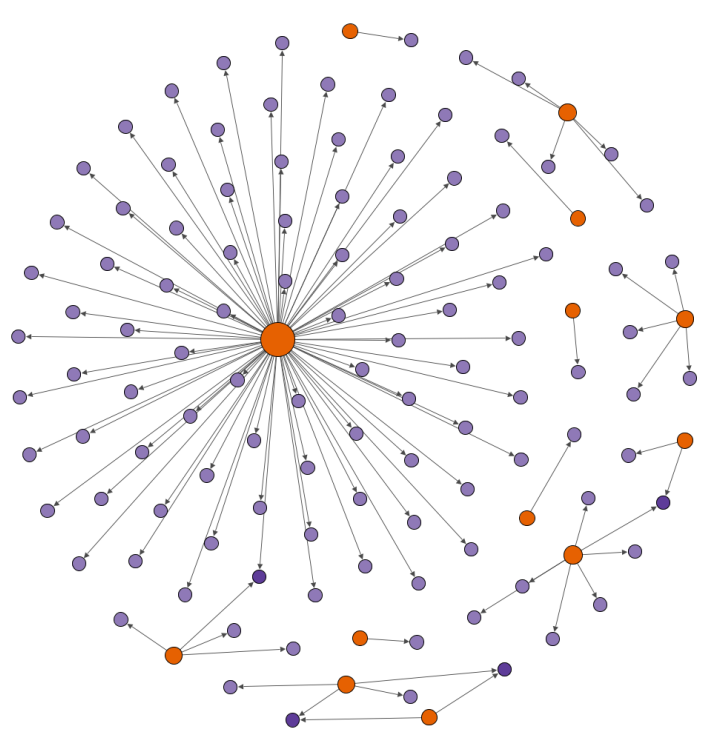

Figure 2: Naming graph based on the intersection of the mentions and the naming data. Larger node size indicates a bigger out degree; Color indicates the in degree (darker nodes were named more times).

cal setting we combine (Ding et al., 2008) and the LIWC lexicon (Tausczik and Pennebaker, 2010). In the statistical setting we use NaSent (Socher et al., 2013).

The motivation to use both methods is twofold: first - while statistical models are generally more robust, accurate and sensitive to context, they require parsing of the processed sentences. Parsing our data is often problematic due to the noise introduced by the OCR algorithm due to the poor quality of the documents (see Figure 1). We expected the lexicon-based method to be more tolerant to noisy or ill-structured sentences. We opted for the LIWC since it offers an array of sentiment and psychological categories that might be relevant in the analysis of such data. 


\begin{tabular}{|c|c|c|}
\hline & Stanford & LIWC \\
\hline Pos & 75 & 292 \\
\hline Neg & 254 & 37 \\
\hline
\end{tabular}

Table 2: Confusion matrix for Stanford and LIWC sentiment algorithms.

Aggregated Sentiment A name may be mentioned a number of times in a single hearing, each time with a different sentiment type or polarity. The aggregated sentiment weight of a witness $i$ toward a mentioned name $j$ is computed as follows:

$$
\operatorname{sentiment}(i, j)=\max _{c \in C A T} \frac{\sum_{k \in U_{i j}} \operatorname{score}\left(u_{i j}^{k}, c\right)}{\left|U_{i j}\right|}
$$

Where $C A T$ is the set of categories used by LIWC or Stanford Sentiment and $U_{i j}$ is the set of all utterances (dialogue acts) in which witness $i$ mentions the name $j$. The score() function is defined slightly different for each setting. In the LIWC setting we define score as:

$$
\operatorname{score}\left(u_{i j}^{k}, c\right)=\frac{\left|\left\{w \in u_{i j}^{k} \mid w \in c\right\}\right|}{\left|u_{i j}^{k}\right|}
$$

In the statistical setting, Stanford Sentiment returns a sentiment category and a weight, we therefore use:

$$
\text { score }\left(u_{i j}^{k}, c\right)= \begin{cases}w_{c}, & \text { if sentiment found } \\ 0, & \text { if c was not returned }\end{cases}
$$

Unfortunately, both approaches to sentiment analysis were not as useful as expected. Most graph edges did not have any sentiment label, either due to the limited sentiment lexicon of the LIWC or due to the noise induced in the OCR process, preventing the Stanford Sentiment engine from parsing many of the sentences. Interestingly, the two approaches did not agree on most sentences (or dialogue acts). The sentiment confusion matrix is presented in Table 2, illustrating the challenge posed by the data.

\subsection{Psychological Categories}

The LIWC lexicon contains more than just positive/negative categories. Table 3 presents a sample of LIWC categories and associated tokens. Figure 3 presents the frequencysave in which each psychological category is used by friendly and uncooperative witnesses. While the Pronoun category is equally used by both parties, the uncooperative witnesses tend to use the I, Self and You categories while the friendly witnesses tend to use the Other and Social. A somewhat surprising result is that the Tentat category is used more by friendly witnesses - presumably reflecting their discomfort with their position as informers.

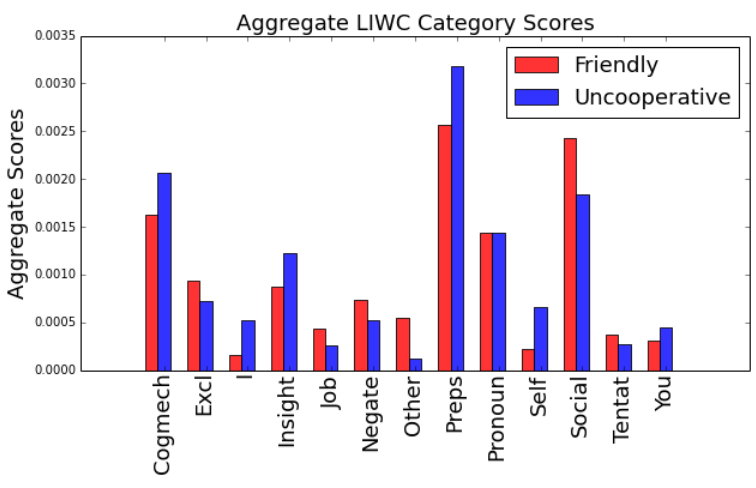

Figure 3: Frequencies of selected LIWC categories in friendly vs. uncooperative testimonies.

\begin{tabular}{|c||l|}
\hline Category & \multicolumn{1}{c|}{ Typical Words } \\
\hline Cogmech & abandon, accept, avoid, admit, know, question \\
\hline Excl & although, besides, but, except \\
\hline I & I, I'd, I'll, I'm, I've, me, mine, my, myself \\
\hline Insight & accept, acknowledge, conclude, know, rational \\
\hline job & work, position, benefit, duty \\
\hline Negate & no, nope, nothing, neither, never, isn't , can't \\
\hline Other & he, him, herself, them \\
\hline Preps & about, against, along, from, outside, since \\
\hline Pronouns & I, anybody, anyone, something, they, you \\
\hline Self & I, mine, ours, myself, us \\
\hline Social & acquaintance, admit, party, comrade, confess, friend, human \\
\hline Tentat & ambiguous, tentative, undecided, depend, hesitant, guess \\
\hline You & thou, thoust, thy, y'all, ya, ye, you, you'd \\
\hline
\end{tabular}

Table 3: LIWC categories and examples of typical words

\section{Conclusion and Future Work}

In this short paper we take a computational approach in analyzing a collection of HUAC hearings. We combine Natural Language Processing and Network Science techniques in order to gain a better understanding of the social dynamics within the entertainment industry in its darkest time. While sentiment analysis did not prove as useful as expected, analysis of network structures and the language usage in an array of psychological dimensions reveals differences between friendly and uncooperative witnesses.

Future work should include a better preprocessing of the data, which is also expected to improve the sentiment analysis. In future work we will analyze the language use in a finer granularity of witness categories, such as the ideological informer, the naive informer and the vindictive informer. We also hope to expand the hearings corpora to include testimonies from more years.

\section{References}

Sibel Adali, Fred Sisenda, and Malik Magdon-Ismail. 2012. Actions speak as loud as words: Predicting 
relationships from social behavior data. In Proceedings of the 21st international conference on World Wide Web, pages 689-698. ACM.

Lada A Adamic, Jun Zhang, Eytan Bakshy, and Mark S Ackerman. 2008. Knowledge sharing and yahoo answers: everyone knows something. In Proceedings of the 17th international conference on World Wide Web, pages 665-674. ACM.

Jiang Bian, Yandong Liu, Ding Zhou, Eugene Agichtein, and Hongyuan Zha. 2009. Learning to recognize reliable users and content in social media with coupled mutual reinforcement. In Proceedings of the 18th international conference on World Wide Web, pages 51-60. ACM.

Cristian Danescu-Niculescu-Mizil and Lillian Lee. 2011. Chameleons in imagined conversations: A new approach to understanding coordination of linguistic style in dialogs. In Proceedings of the Workshop on Cognitive Modeling and Computational Linguistics, ACL 2011.

Cristian Danescu-Niculescu-Mizil, Michael Gamon, and Susan Dumais. 2011. Mark my words! Linguistic style accommodation in social media. In Proceedings of $W W W$, pages 745-754.

Cristian Danescu-Niculescu-Mizil, Lillian Lee, Bo Pang, and Jon Kleinberg. 2012. Echoes of power: Language effects and power differences in social interaction. In Proceedings of $W W W$, pages 699-708.

Xiaowen Ding, Bing Liu, and Philip S. Yu. 2008. A holistic lexicon-based approach to opinion mining. In Proceedings of the 2008 International Conference on Web Search and Data Mining, WSDM '08, pages 231-240, New York, NY, USA. ACM.

Jacob Eisenstein, Brendan O'Connor, Noah A Smith, and Eric P Xing. 2010. A latent variable model for geographic lexical variation. In Proceedings of the 2010 Conference on Empirical Methods in Natural Language Processing, pages 1277-1287. Association for Computational Linguistics.

Jenny Rose Finkel, Trond Grenager, and Christopher Manning. 2005. Incorporating non-local information into information extraction systems by gibbs sampling. In Proceedings of the 43rd Annual Meeting on Association for Computational Linguistics, pages 363-370. Association for Computational Linguistics.

Yoav Goldberg and Jon Orwant. 2013. Syntacticngrams over time from a very large corpus of english books. In Second Joint Conference on Lexical and Computational Semantics.

Heeyoung Lee, Yves Peirsman, Angel Chang, Nathanael Chambers, Mihai Surdeanu, and Dan Jurafsky. 2011. Stanford's multi-pass sieve coreference resolution system at the conll-2011 shared task. In Proceedings of the Fifteenth Conference on
Computational Natural Language Learning: Shared Task, pages 28-34. Association for Computational Linguistics.

Victor S Navasky. 2003. Naming Names: With a New Afterword by the Author. Macmillan.

Brendan O'Connor, Jacob Eisenstein, Eric P Xing, and Noah A Smith. 2010. A mixture model of demographic lexical variation. In Proceedings of NIPS workshop on machine learning in computational social science, pages 1-7.

Daniel M Romero, Brendan Meeder, and Jon Kleinberg. 2011. Differences in the mechanics of information diffusion across topics: idioms, political hashtags, and complex contagion on twitter. In Proceedings of the 20th international conference on World wide web, pages 695-704. ACM.

Richard Socher, Alex Perelygin, Jean Wu, Jason Chuang, Christopher D. Manning, Andrew Y. Ng, and Christopher Potts. 2013. Recursive deep models for semantic compositionality over a sentiment treebank. In Proceedings of the 2013 Conference on Empirical Methods in Natural Language Processing, pages 1631-1642, Stroudsburg, PA, October. Association for Computational Linguistics.

Yla R. Tausczik and James W. Pennebaker. 2010. The Psychological Meaning of Words: LIWC and Computerized Text Analysis Methods. Journal of Language and Social Psychology, 29(1):24-54, March. 